\title{
Two-sided Matching Decision under Multi-granularity Uncertain Linguistic Environment
}

\author{
Qi Yue ${ }^{1}$, Yongshan Peng ${ }^{2}$, Bingwen $\mathrm{Yu}^{3}, \mathrm{Yu} \mathrm{Hong}^{4} *$ and Quan Xiao ${ }^{5}$ \\ ${ }^{1}$ School of Information Management, Jiangxi University of Finance and \\ Economics, Nanchang, China \\ ${ }^{2}$ School of Physical Education, Jiangxi University of Finance and Economics, \\ Nanchang 330013, China \\ ${ }^{3}$ School of Economics, Jiangxi University of Finance and Economics, Nanchang \\ 330013, China \\ ${ }^{4}$ College of Humanities and Sciences, Northeast Normal University, Changchun \\ 130117, China \\ ${ }^{4}$ School of Information Management, Jiangxi University of Finance and \\ Economics, Nanchang, China \\ yueqichina@126.com,pengyongshanjxcd@sina.com,yubingwen2005@163.com, \\ *acjlhong@126.com,xiaoquan@foxmail.com
}

\begin{abstract}
With respect to the two-sided matching problem, where the agents' preferences are in the format of multi-granularity uncertain linguistic terms, a matching method is proposed. Some basic concepts and definitions on uncertain linguistic term and 2-tuple are firstly given. Based on this, the two-sided matching problem with multi-granularity uncertain linguistic terms is described. Then, multi-granularity uncertain linguistic term matrixes are transformed into multi-granularity 2-tuple matrixes. Furthermore, a multi-objective optimization model is developed by using the extended 2-tuple weighted average. By using the normalization method and the linear weighted method twice, the multi-objective optimization model can be converted into a single-objective optimization model. By solving the model, the matching alternative can be obtained. Example of matching demand and supply in agricultural knowledge illustrates the feasibility and effectiveness of this method.
\end{abstract}

Keywords: two-sided matching; multi-granularity; uncertain linguistic term; 2-tuple; optimization model

\section{Introduction}

The two-sided matching problems widely exist in the real world. Examples contain the stable marriage problem [1-4], the CEOs selection problem [5-8], the college admission problem [9-12], the employee selection problem [13-15], and the personnel assignment problem [16-18]. Since the reasonable and effective matching alternative has a promoting effect for improving the efficiency of economic management activities and the satisfaction degrees of two-sided agents, studying on the two-sided matching problems has important theoretical significance and practical application value.

There are plenty of two-sided matching problems in many fields of real life, such as marriage assignment [1], college admission [2], employee selection [3], personnel assignment [4] and CEOs to companies [5], Therefore two-sided matching is a research topic with extensive application backgrounds.

At present, the two-sided matching problems with different forms of information have attracted extensively attentions. For example, Gale and Shapley initially investigate the concept, existence, optimality and algorithm of stable assignment [6]. The notions of 
vonNeumann-Morgenstern (vNM) farsightedly stable sets are adopted to determine which matchings are possibly stable when agents are farsighted in one-to-one matching problems [7]. Boon and Sierksma match position with player in soccer team formation using linear optimization models, [8]. Sethuraman et al. focus on the geometric structure of fractional stable matchings in the stable admission problem [9]. Ehlers studies truncation strategies in matching markets using the deferred acceptance algorithm, and show that truncation strategies are also applicable to all priority mechanisms and all linear programming mechanisms [10]. Uetake and Watanabe propose an approach to estimate a nontransferable utility in two-sided matching models [11].

The existing studies develop the methods for solving the two-sided matching problems, and expand the actual application background. However, due to the complexity of actual problems, the ambiguity of thinking judgement, the preferences provided by agents are in the format of uncertain linguistic terms, and the granularity of the linguistic term sets is also different. The existing studies seldom consider this case. Therefore, how to consider the two-sided matching problem with multi-granularity uncertain linguistic terms is a valuable research topic. In this paper, a novel two-sided matching method is presented.

The remainder of this paper is arranged as follows: Section 2 introduces some concepts of uncertain linguistic term and 2-tuple. Section 3 formulates the two-sided matching problem with multi-granularity uncertain linguistic terms. Section 4 proposes a new method. Section 5 gives a matching example in agricultural knowledge. Section 6 summarizes the main features of the proposed method.

\section{The Concepts}

In this section, some concepts on uncertain linguistic term and 2-tuple are given.

Definition 1. Let be the set of linguistic terms, and $z^{*}$ be the set of positive integers. An uncertain linguistic term $\tilde{s}$ is expressed by $\tilde{s}=\left\{s^{L}, s^{L+1}, \cdots, s^{U}\right\}$, where $s^{L}, s^{L+1}, \cdots, s^{U} \in S, L, U \in Z^{*}, L \leq U$. Here $s^{L}$ and $s^{U}$ are the lower bound and upper bound of $\tilde{s}$, respectively. Specially, if $s^{L}=s^{U}$, then $\tilde{s}$ reduces to a linguistic term.

By Definition 1, we know that $\tilde{s}$ is a discrete set. For simplicity, we express $\tilde{s}$ as $\tilde{s}=\left[s^{L}, s^{U}\right]$. In the considered two-sided matching problem, if $\tilde{r}=\left[r^{L}, r^{U}\right]$ is an uncertain linguistic term, then linguistic terms $r^{L}, r^{L+1}, \ldots, r^{U-1}$ and $r^{U}$ possess the same possibility.

Definition 2. Let $\tilde{r}$ be an uncertain linguistic term, then the probability vector on $\tilde{r}$ is expressed by $\tilde{p}_{\tilde{r}}=\left(p_{\tilde{r}}^{L}, \cdots, p_{\tilde{r}}^{k}, \cdots, p_{\dot{r}}^{U}\right)$, where $p_{r}^{k}=1 /(U-L+1)$.

Definition 3 [12-15]. Let $S=\left\{s_{0}, s_{1}, \cdots, s_{g}\right\}$ be a linguistic term set and $\beta \in[0, g]$ a value supporting the result of a symbolic aggregation operation, then the 2-tuple that expresses the equivalent information to $\beta$ is obtained with the following function $\theta$ :

$$
\begin{gathered}
\theta:[0, g] \rightarrow S \times[-0.5,0.5) \\
\theta(\beta)=\left(s_{i}, \alpha\right)= \begin{cases}s_{i}, & i=\operatorname{round}(\beta) \\
\alpha=\beta-i, & \alpha \in[-0.5,0.5)\end{cases}
\end{gathered}
$$

where round is the usual rounding operation, and $s_{i}$ has the closest index label to $\beta$, and $\alpha$ is the value of the symbolic translation.

Definition 4 [12-15]. Let $S=\left\{s_{0}, s_{1}, \cdots, s_{g}\right\}$ be a linguistic term set and $\left(s_{i}, \alpha\right)$ be a 2 tuple. There is always a function $\theta^{-1}$, such that, from a 2-tuple it returns its equivalent numerical value $\beta \in[0, g]$ : 


$$
\begin{gathered}
\theta^{-1}: S \times[-0.5,0.5) \rightarrow[0, g] \\
\theta^{-1}\left(s_{i}, \alpha\right)=i+\alpha=\beta
\end{gathered}
$$

Remark 1: From Definitions 3 and 4, it is obvious that the conversion of a linguistic term into a 2-tuple consists of adding a value 0 as symbolic translation, i.e., $s_{i} \in S \Rightarrow\left(s_{i}, 0\right)$.

Definition 5 [12-15]. Let $x=\left\{\left(r_{1}, \alpha_{1}\right),\left(r_{2}, \alpha_{2}\right), \cdots,\left(r_{f}, \alpha_{f}\right)\right\}$ be a set of 2-tuples and $w=\left\{w_{1}, w_{2}, \cdots, w_{f}\right\}$ be their associated weights, where $w_{i} \in[0,1]$ and $\sum w_{i}=1$. The 2tuple weighted average is defined as $\left.\bar{r}^{w}=\theta \mid \frac{\left(\sum_{c=1}^{f} \theta^{-1}\left(r_{c}, \alpha_{c}\right) w_{c}\right.}{\sum_{c=1}^{f} w_{c}}\right\}$, where $\theta$ is a symbolic translation, and $\theta^{-1}$ is the inverse of $\theta$.

Definition 6. Let $\tilde{r}=\left[r^{L}, r^{U}\right]$ be an uncertain linguistic term, and $\tilde{p}_{\tilde{r}}$ be the probability vector on $\tilde{r}$, then by Definitions 2 and 3, the expectation of $\tilde{r}$ (noted as $E(\tilde{r})$ ) is calculated by

$$
E(\tilde{r})=\theta\left(\sum_{k=r^{L}}^{r^{U}} \frac{\theta^{-1}(k, 0)}{U-L+1}\right)
$$

In order to deal with 2-tuples, the definition of the extended 2-tuple weighted average should be given according to Definitions 5 .

Definition 7. Let $x=\left\{\left(r_{1}, \alpha_{1}\right),\left(r_{2}, \alpha_{2}\right), \cdots,\left(r_{f}, \alpha_{f}\right)\right\}$ be a set of 2-tuples and $w=\left\{w_{1}, w_{2}, \cdots, w_{f}\right\}$ be their associated weights, where $w_{i} \in[0,1]$ and $\sum w_{i} \in[0,1]$. The extended 2-tuple weighted average is defined by

$$
\tilde{\bar{r}}^{w}=\left\{\begin{array}{l}
\left|\begin{array}{c}
\left.\mid \sum_{c=1}^{f} \theta^{-1}\left(r_{c}, \alpha_{c}\right) w_{c}\right) \\
|f|
\end{array}\right|, \quad 0<\sum_{c=1}^{f} w_{c} \leq 1 \\
\mid \theta\left(\sum_{c=1}^{f} w_{c} \theta^{-1}\left(r_{c}, \alpha_{c}\right) w_{c}\right)=\theta(0), \quad \sum_{c=1}^{f} w_{c}=0
\end{array}\right.
$$

\section{The Problem}

This paper considers the two-sided matching problem, where the agents' preferences are in the format of multi-granularity uncertain linguistic terms. Let $A=\left\{A_{1}, A_{2}, \cdots, A_{m}\right\}$ $(m \geq 2)$ be the set of agents of side $A$, where $A_{i}$ denotes the $i$ th agent of side $A$; $B=\left\{B_{1}, B_{2}, \cdots, B_{n}\right\} \quad(n \geq m \geq 2)$ be the set of agents of side $B$, where $B_{j}$ denotes the $j$ th agent of side $B$. Let $S_{A_{i}}=\left\{s_{0}, s_{1}, \cdots, s_{p_{i}}\right\}$ be the set of satisfaction linguistic terms given by $A_{i}$, where $p_{i}+1$ denotes the cardinality of set $S_{A_{i}} ; S_{B_{j}}=\left\{s_{0}, s_{1}, \cdots, s_{q_{j}}\right\}$ be the set of satisfaction linguistic terms given by $B_{j}$, where $q_{j}+1$ denotes the cardinality of set $S_{B_{j}}$. Let $\tilde{L}_{A}=\left[\tilde{l}_{i j}^{A}\right]_{m \times n}$ be the multi-granularity uncertain linguistic term matrix from side $A$ to $B$, where $\tilde{l}_{i j}^{A}=\left[l_{i j}^{A L}, l_{i j}^{A U}\right]$ denotes the multi-granularity uncertain linguistic term for agent 
$A_{i}$ towards $B_{j}, l_{i j}^{A L}, l_{i j}^{A U} \in S_{A_{i}} ; \tilde{L}_{B}=\left[\tilde{l}_{i j}^{B}\right]_{m \times n}$ be the multi-granularity uncertain linguistic term matrix from side $B$ to $A$, where $\tilde{l}_{i j}^{B}=\left[l_{i j}^{B L}, l_{i j}^{B U}\right]$ denotes the multi-granularity uncertain linguistic term for agent $B_{j}$ towards $A_{i}, l_{i j}^{B L}, l_{i j}^{B U} \in S_{B_{j}}$. Let $\mu$ be the two-sided matching or matching alternative, $\mu=\mu_{T} \cup \mu_{o}$. Here, $\mu_{T}$ denotes the set of matching pairs, $\mu_{T}=\left\{\left(A_{i}, B_{\sigma(i)}\right) \mid i=1, \cdots, m\right\}$, where $\sigma(1), \cdots, \sigma(m)$ is the permutation of $1,2, \cdots, m$; $\mu_{o}$ denotes the set of single pairs, $\mu_{o}=\left\{\left(B_{j}, B_{j}\right) \mid j \in\{1, \cdots, n\} \backslash\{\sigma(1), \cdots, \sigma(m)\}\right\}$.

The considered problem is how to obtain the reasonable matching alternative based on multi-granularity uncertain linguistic term matrixes $\tilde{L}_{A}=\left[\tilde{l}_{i j}^{A}\right]_{m \times n}$ and $\tilde{L}_{B}=\left[\tilde{l}_{i j}^{B}\right]_{m \times n}$.

\section{The Method}

This section will present a matching method for solving the two-sided matching problem with multi-granularity uncertain linguistic terms.

Firstly, by Eqs. (1a)-(3), we transform multi-granularity uncertain linguistic term matrixes $\tilde{L}_{A}=\left[\tilde{l}_{i j}^{A}\right]_{m \times n}$ and $\tilde{L}_{B}=\left[\tilde{l}_{i j}^{B}\right]_{m \times n}$ into multi-granularity 2-tuple matrixes $L_{A}=\left[\left(l_{i j}^{A}, \alpha_{i j}\right)\right]_{m \times n}$ and $L_{B}=\left[\left(l_{i j}^{B}, \beta_{i j}\right)\right]_{m \times n}$, where multi-granularity 2-tuples $\left(l_{i j}^{A}, \alpha_{i j}\right)$ and $\left(l_{i j}^{B}, \beta_{i j}\right)$ are calculated by

$$
\begin{aligned}
& \left(l_{i j}^{A}, \alpha_{i j}\right)=\theta\left(\frac{\theta^{-1}\left(l_{i j}^{A L}, 0\right)+\theta^{-1}\left(l_{i j}^{A U}, 0\right)}{2}\right) \\
& \left(l_{i j}^{B}, \beta_{i j}\right)=\theta\left(\frac{\theta^{-1}\left(l_{i j}^{B L}, 0\right)+\theta^{-1}\left(l_{i j}^{B U}, 0\right)}{2}\right)
\end{aligned}
$$

Based on multi-granularity 2-tuple matrixes $L_{A}$ and $L_{B}$, we consider to construct an optimization model for obtaining the matching alternative considering matching constraints. On the one hand, according to the characteristics of linguistic term and 2-tuple, we know that the greater $l_{i j}^{A}$ or $l_{i j}^{B}$ is, the greater satisfaction degree of agent $A_{i}$ or $B_{j}$ is. Hence, we take the maximization 2-tuple as the objective function. On the other hand, due to $m \leq n$, the matching constraints can be interpreted as follows: agent $A_{i}$ has exactly one matching agent of side $B$ (i.e., $\sum_{j=1}^{n} x_{i j}=1$ ), and agent $B_{j}$ has at most one matching agent of side $A$ (i.e., $\left.\sum_{i=1}^{m} x_{i j} \leq 1\right)$, where $x_{i j}=\left\{\begin{array}{ll}0, & \mu\left(A_{i}\right) \neq B_{j} \\ 1, & \mu\left(A_{i}\right)=B_{j}\end{array}\right.$. Moreover, by using the extended 2tuple weighted average, the following multi-objective optimization model (7) can be set up:

$$
\begin{array}{ll}
\max & Z\left(A_{i}\right)=\theta\left(\sum_{j=1}^{n} \theta^{-1}\left(l_{i j}^{A}, \alpha_{i j}\right) x_{i j}\right), i=1,2, \cdots, m \\
\max & Z\left(B_{j}\right)=\theta\left(\sum_{i=1}^{m} \theta^{-1}\left(l_{i j}^{B}, \beta_{i j}\right) x_{i j}\right), j=1,2, \cdots, n \\
\text { s.t. } & \sum_{j=1}^{n} x_{i j}=1, i=1,2, \cdots, m
\end{array}
$$




$$
\begin{aligned}
& \sum_{i=1}^{m} x_{i j} \leq 1, j=1,2, \cdots, n \\
& x_{i j}=0 \text { or } 1, i=1,2, \cdots, m, j=1,2, \cdots, n
\end{aligned}
$$

In order to solve model (7), considering that symbolic translation $\theta$ is a monotone increasing function, the optimal solution(s) of the following optimization model (8) is that of model (7):

$$
\begin{array}{ll}
\max & Z\left(A_{i}\right)=\sum_{j=1}^{n} \theta^{-1}\left(l_{i j}^{A}, \alpha_{i j}\right) x_{i j}, i=1,2, \cdots, m \\
\max \quad Z\left(B_{j}\right)=\sum_{i=1}^{m} \theta^{-1}\left(l_{i j}^{B}, \beta_{i j}\right) x_{i j}, j=1,2, \cdots, n \\
\text { s.t. } \quad \sum_{j=1}^{n} x_{i j}=1, i=1,2, \cdots, m \\
\quad \sum_{i=1}^{m} x_{i j} \leq 1, j=1,2, \cdots, n \\
\quad x_{i j}=0 \text { or } 1, i=1,2, \cdots, m, j=1,2, \cdots, n
\end{array}
$$

In order to solve model (8), considering that the cardinalities of sets $S_{A_{i}}(i=1,2, \cdots, m)$ may be not equal, we should normalize Eqs. (8a) and (8b) firstly. Usually, the status of each agent of one side is the same. Hence, we can suppose that each agent of one side has equal priority. Then, using the linear weighted method, model (8) can be transformed into the following bi-objective optimization model (9):

$$
\begin{aligned}
& \max \quad Z(A)=\frac{1}{m} \sum_{i=1}^{m} \frac{\sum_{j=1}^{n} \theta^{-1}\left(l_{i j}^{A}, \alpha_{i j}\right) x_{i j}}{p_{i}+1} \\
& \max \quad Z(B)=\frac{1}{n} \sum_{j=1}^{n} \frac{\sum_{i=1}^{m} \theta^{-1}\left(l_{i j}^{B}, \beta_{i j}\right) x_{i j}}{q_{j}+1} \\
& \text { s.t. } \quad \sum_{j=1}^{n} x_{i j}=1, i=1,2, \cdots, m \\
& \quad \sum_{i=1}^{m} x_{i j} \leq 1, j=1,2, \cdots, n \\
& \quad x_{i j}=0 \text { or } 1, i=1,2, \cdots, m, j=1,2, \cdots, n
\end{aligned}
$$

In order to solve model (9), the linear weighted method is also used. Let $w_{A}$ and $w_{B}$ be the weight of objectives functions $\mathrm{Z}(\mathrm{A})$ and $\mathrm{Z}(\mathrm{B})$, respectively, such that $w_{A}, w_{B} \in[0,1]$, $w_{A}+w_{B}=1$, then model (9) can be transformed into the following single-objective optimization model (10): 


$$
\begin{array}{ll}
\max & Z=\left\{\frac{w_{A}}{m} \sum_{i=1}^{m} \frac{\sum_{j=1}^{n} \theta^{-1}\left(l_{i j}^{A}, \alpha_{i j}\right)}{p_{i}+1}+\left.\frac{w_{B}}{n} \sum_{j=1}^{n} \frac{\sum_{i=1}^{m} \theta^{-1}\left(l_{i j}^{B}, \beta_{i j}\right)}{q_{j}+1}\right|_{i j}\right. \\
\text { s.t. } & \sum_{j=1}^{n} x_{i j}=1, i=1,2, \cdots, m \\
& \sum_{i=1}^{m} x_{i j} \leq 1, j=1,2, \cdots, n \\
& x_{i j}=0 \text { or } 1, i=1,2, \cdots, m, j=1,2, \cdots, n
\end{array}
$$

Model (10) can be solved by the existing mathematical optimization software. According to multiple-objective programming theory, the optimal solution of model (10) is the efficient solution of model (9). Then the matching alternative can be obtained based on the obtained optimal solution.

In sum, an algorithm is developed to solve the two-sided matching problem with multigranularity uncertain linguistic terms as follows:

Step 1. Transform multi-granularity uncertain linguistic term matrixes $\tilde{L}_{A}=\left[\tilde{l}_{i j}^{A}\right]_{m \times n}$ and $\tilde{L}_{B}=\left[\tilde{i}_{i j}^{B}\right]_{m \times n}$ into multi-granularity 2-tuple matrixes $L_{A}=\left[\left(l_{i j}^{A}, \alpha_{i j}\right)\right]_{m \times n}$ and $L_{B}=\left[\left(l_{i j}^{B}, \beta_{i j}\right)\right]_{m \times n}$ by Eqs (5) and (6).

Step 2. Built the multiple-objective optimization model (7) based on multi-granularity 2-tuple matrixes $L_{A}$ and $L_{B}$ by Eq. (4).

Step 3. Transform model (7) into model (8).

Step 4. Transform model (8) into model (9) by using the normalization method and the linear weighted method.

Step 5. Transform model (9) into model (10) by using the linear weighted method.

Step 6. Obtain the matching alternative by solving model (10).

\section{The Example}

The agricultural innovation intermediary in high-tech zone of Nanchang plans to make agricultural knowledge matchings between end-users of agricultural entrepreneurs (demand side) and producers of agricultural R\&D and KIBS (supply side). Four agricultural entrepreneurs $A_{1}, A_{2}, \ldots, A_{4}$ and six producers $B_{1}, B_{2}, \ldots, B_{6}$ participate in the process of matching. Agricultural entrepreneur $A_{i}$ evaluates producers from market prospect, complexity, and price. Producer $B_{j}$ evaluates agricultural entrepreneurs from income, conversion speed, and technological level. The satisfaction linguistic term sets $S_{A_{1}}=S_{A_{2}}=\left\{s_{0}=\mathrm{VL}(\right.$ Very low $), s_{1}=\mathrm{L}(\mathrm{Low}), s_{2}=\mathrm{ML}($ Medium low $), s_{3}=\mathrm{M}($ Medium $)$, $s_{4}=\mathrm{MH}($ Medium high $), s_{5}=\mathrm{H}($ High $), s_{6}=\mathrm{VH}($ Very high $\left.)\right\}, s_{A_{3}}=S_{A_{4}}=\left\{s_{0}=\mathrm{VL}\right.$ (Very low),

$s_{1}=\mathrm{L}($ Low $), s_{2}=\mathrm{M}($ Medium $), s_{3}=\mathrm{H}($ High $), s_{4}=\mathrm{VH}($ Very high $\left.)\right\}$. The satisfaction linguistic term set $S_{B_{1}}=S_{B_{2}}=S_{B_{3}}=S_{B_{4}}=\left\{s_{0}=\mathrm{VL}\right.$ (Very low), $s_{1}=\mathrm{L}($ Low $), s_{2}=\mathrm{M}$ (Medium), $s_{3}=\mathrm{H}($ High $), \quad s_{4}=\mathrm{VH}($ Very high $\left.)\right\}, \quad S_{B_{5}}=S_{B_{6}}=\left\{s_{0}=\mathrm{L}(\right.$ low $), \quad s_{1}=\mathrm{M}($ Medium $)$, 
$s_{2}=\mathrm{H}($ High $\left.)\right\}$. The multi-granularity uncertain linguistic term matrixes $\tilde{L}_{A}=\left[\tilde{l}_{i j}^{A}\right]_{4 \times 6}$ and $\tilde{L}_{B}=\left[\tilde{l}_{i j}^{B}\right]_{4 \times 6}$ are provided as follows.

$$
\begin{aligned}
\tilde{L}_{A}= & {\left[\begin{array}{llllll}
{\left[s_{2}, s_{3}\right]} & {\left[s_{3}, s_{4}\right]} & {\left[s_{2}, s_{2}\right]} & {\left[s_{4}, s_{5}\right]} & {\left[s_{1}, s_{2}\right]} & {\left[s_{5}, s_{6}\right]} \\
{\left[s_{3}, s_{4}\right]} & {\left[s_{2}, s_{3}\right]} & {\left[s_{1}, s_{1}\right]} & {\left[s_{0}, s_{1}\right]} & {\left[s_{4}, s_{4}\right]} & {\left[s_{2}, s_{2}\right]} \\
{\left[s_{2}, s_{2}\right]} & {\left[s_{0}, s_{1}\right]} & {\left[s_{1}, s_{2}\right]} & {\left[s_{3}, s_{4}\right]} & {\left[s_{2}, s_{3}\right]} & {\left[s_{4}, s_{4}\right]} \\
{\left[s_{1}, s_{2}\right]} & {\left[s_{4}, s_{4}\right]} & {\left[s_{3}, s_{4}\right]} & {\left[s_{0}, s_{1}\right]} & {\left[s_{2}, s_{3}\right]} & {\left[s_{2}, s_{2}\right]}
\end{array}\right] } \\
\tilde{L}_{B}= & {\left[\begin{array}{llllll}
{\left[s_{2}, s_{3}\right]} & {\left[s_{3}, s_{4}\right]} & {\left[s_{2}, s_{2}\right]} & {\left[s_{3}, s_{3}\right]} & {\left[s_{1}, s_{1}\right]} & {\left[s_{0}, s_{1}\right]} \\
{\left[s_{3}, s_{4}\right]} & {\left[s_{2}, s_{3}\right]} & {\left[s_{1}, s_{1}\right]} & {\left[s_{2}, s_{3}\right]} & {\left[s_{1}, s_{2}\right]} & {\left[s_{2}, s_{2}\right]} \\
{\left[s_{2}, s_{2}\right]} & {\left[s_{0}, s_{1}\right]} & {\left[s_{2}, s_{3}\right]} & {\left[s_{1}, s_{2}\right]} & {\left[s_{0}, s_{1}\right]} & {\left[s_{1}, s_{2}\right]} \\
{\left[s_{1}, s_{2}\right]} & {\left[s_{2}, s_{2}\right]} & {\left[s_{3}, s_{4}\right]} & {\left[s_{4}, s_{4}\right]} & {\left[s_{2}, s_{2}\right]} & {\left[s_{1}, s_{2}\right]}
\end{array}\right] }
\end{aligned}
$$

To determine the reasonable matching alternative, a brief description of the matching process is given below. Firstly, by Eqs. (5) and (6), multi-granularity uncertain linguistic term matrixes $\tilde{L}_{A}=\left[\tilde{l}_{i j}^{A}\right]_{4 \times 6}$ and $\tilde{L}_{B}=\left[\tilde{l}_{i j}^{B}\right]_{4 \times 6}$ are transformed into multi-granularity 2-tuple matrixes $L_{A}=\left[\left(l_{i j}^{A}, \alpha_{i j}\right)\right]_{4 \times 6}$ and $L_{B}=\left[\left(l_{i j}^{B}, \beta_{i j}\right)\right]_{4 \times 6}$. Then, the multiple-objective optimization model (7) can be built by Eq. (4). By using the normalization method and the linear weighted method, model (7) can be transformed into model (9). Suppose $w_{A}=0.6$ and $w_{B}=0.4$, model (9) can be transformed into model (10), where coefficient matrix $C=\left(c_{i j}\right)_{4 \times 6}$ is calculated by

$$
C=\left[\begin{array}{cccccc}
0.0869 & 0.1217 & 0.0695 & 0.1364 & 0.0544 & 0.129 \\
0.1217 & 0.0869 & 0.0348 & 0.044 & 0.119 & 0.0873 \\
0.0867 & 0.0217 & 0.0783 & 0.125 & 0.0861 & 0.1533 \\
0.065 & 0.1467 & 0.145 & 0.0683 & 0.1194 & 0.0933
\end{array}\right]
$$

Lastly, by solving model (10), the matching alternative $\mu=\mu_{T}^{*} \cup \mu_{o}^{*}$ can be obtained, i.e.,

$$
X^{*}=\left[x_{i j}^{*}\right]_{4 \times 6}=\left[\begin{array}{cccccc}
0 & 0 & 0 & 1 & 0 & 0 \\
1 & 0 & 0 & 0 & 0 & 0 \\
0 & 0 & 0 & 0 & 0 & 1 \\
0 & 1 & 0 & 0 & 0 & 0
\end{array}\right]
$$

According to the unique optimal solution $X^{*}$, the matching alternative $\mu^{*}$ can be obtained, i.e., $\mu^{*}=\mu_{T}^{*} \cup \mu_{o}^{*} \quad$, where $\mu_{T}^{*}=\left\{\left(A_{1}, B_{4}\right),\left(A_{2}, B_{1}\right),\left(A_{3}, B_{6}\right),\left(A_{4}, B_{2}\right)\right\}$, $\mu_{o}^{*}=\left\{\left(B_{3}, B_{3}\right),\left(B_{5}, B_{5}\right)\right\}$. Hence, entrepreneur $A_{1}$ matches with producer $B_{4}$, entrepreneur $A_{2}$ matches with producer $B_{1}$, entrepreneur $A_{3}$ matches with producer $B_{6}$, entrepreneur $A_{4}$ matches with producer $B_{2}$, producers $B_{3}$ and $B_{5}$ are single.

\section{Conclusion}

A matching method for solving the two-sided matching problem with multi-granularity uncertain linguistic terms is proposed. Comparing with the existing methods, the proposed method has distinct characteristics as discussed below.

Firstly, the related concepts of uncertain linguistic term and 2-tuple are given. It is a beneficial supplement of theory of linguistic term. Secondly, a multi-objective 
optimization model is constructed. By solving it, it could be easier to obtain the reasonable stable matching alternative. Finally, the proposed method is theoretically sound and computationally simple which provides a new way to solve the considered two-sided matching problem and can be adopted for practical use. When the multi-granularity linguistic terms are incomplete, how to obtain the reasonable matching alternative is worth to study for us in future.

\section{Acknowledgment}

This work was partly supported by the National Natural Science Foundation of China (Project Nos. 71261007, 71261006, 71462015, 71361021, 71363022, 71361011).

\section{References}

[1] R. W. Irving, D. F. Manlove, and G. O’Malley, Journal of Discrete Algorithms, vol. 7, no. 213, (2009).

[2] M. Clark, J. Rothstein, and D. W. Schanzenbach, Econ. Educ. Rev. vol. 28, no. 295, (2009).

[3] K. J. Lauver, and A. Kristof-Brown, J. Vocat. Behav. vol. 59, no. 454, (2001).

[4] A. E. Roth, and E. Peranson, Am. Econ. Rev. vol. 89, no. 748, (1999).

[5] X. Gabaix, and A. Landier, Q. J. Econ. vol. 123, no. 49 (2008).

[6] D. Gale, and L. Shapley, Am. Math. Mon. vol. 69, no. 9 (1962).

[7] A. Mauleon, V. J. Vannetelbosch, and W. Vergote, Theoretical Economics, vol. 6, no. 499, (2011).

[8] B. H. Boon, and G. Sierksma, Eur. J. Oper. Res. vol. 148, no. 277 (2003).

[9] J. Sethuraman, C. P. Teo, and L. Qian, Math. Oper. Res. vol. 31, no. 581, (2006).

[10] L. Ehlers, Math. Oper. Res. vol. 33, no. 327 (2008).

[11] K. Uetake, Y. Watanabe, Econ. Lett. vol. 116, no. 535, (2012).

[12] F. Herrera, and E. Herrera-Viedma, Syst. Man Cy. A. vol. 27, no. 646, (1997)

[13] F. Herrera, E. Herrera-Viedma, and L. Martinez, Fuzzy Set. Syst. vol. 114, no. 43, (2000).

[14] F. Herrera, and L. Martinez, IEEE T. Syst. Man Cy. B. vol. 31, no. 227, (2001).

[15] F. Herrera, and L. Martinez, Syst. Man Cy. B. vol. 31, no. 227, (2001).

\section{Authors}

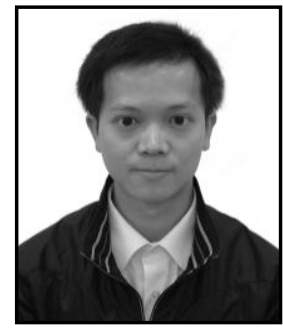

Qi Yue, he was born in 1983. He received the Ph.D. degree in school of business administration from northeastern university, shenyang, China, in 2011. He is currently a lecturer in the school of information management at Jiangxi University of finance and economics. He is the author or coauthor of near forty papers published in international and local journals. His current interests include information science, management science, decision analysis and operations research. E-mail: yueqichina @126.com.

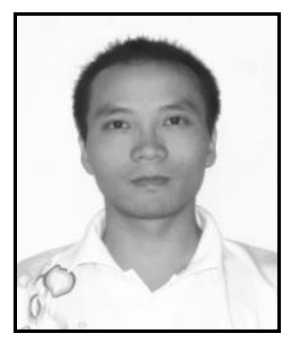

Yongshan Peng, he was born in 1976. He received the Ph.D. degree in school of sports physical education from the normal university of South China, Guangzhou in 2012. He is currently a lecture in the school of sports institute at Jiangxi university of finance and economics. He is the author or coauthor of near thirty papers published in international and local journals. His current interests include sports culture, school physical education, sports management and body culture. E-mail: pengyongshanjxcd@sina.com.

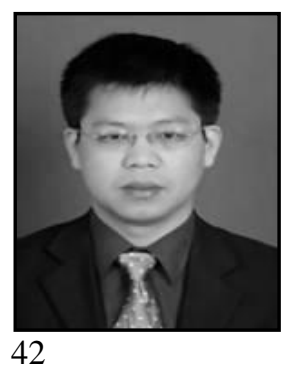

Bing-wen Yu, he was born in 1974. He received the Ph.D. degree in school of business administration from Zhongnan University of Economics and Law, Wuhan, China, in 2011. He is currently a lecturer in the school of economics at Jiangxi university of finance and 


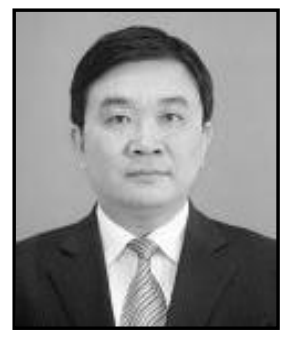

economics. He is the author or coauthor of fifteen papers published in international and local journals. His current interests include industrial economy, appraisal evaluation and real estate research. E-mail: yu_bingwen@sina.cn.

*Yu Hong, he was born in 1971. He received the Ph.D degree in Northeast Asian Studies College from Jilin University, Changchun, China in 2009. He's currently an associate professor in College of Humanities and Sciences, Northeast Normal University, and Jilin University of Finance and Economics. His research interests include world economy, international trade and econometric analysis. He has published two monographs and over forty research papers on international and local journals or conferences. Email: acjlhong@126.com.

Quan Xiao, he was born in 1983. He received the Ph.D degree in School of Management from Huazhong University of Science and Technology, Wuhan, China in 2010. He's currently a lecturer in School of Information Technology at Jiangxi University of Finance and Economics. His research interests include decision support methodologies and systems, risk recognition and information systems. He has published over twenty papers on international and local journals or conferences. Email: xiaoquan@foxmail.com. 
International Journal of $u-$ and e- Service, Science and Technology Vol.8, No. 11 (2015) 